\title{
ESTUDO DE BASES TEÓRICAS PARA PESQUISA SOBRE A DEFINIÇÃO E PROTEÇÃO JURÍDICA DO CONHECIMENTO EM FRANQUIAS
}

\author{
Fernanda C. Frustockl La Rosa ${ }^{1}$ \\ Silvio Bitencourt da Silva ${ }^{2}$
}

\begin{abstract}
Research in the context of interorganizational relations points to knowledge as the most important organizational resource and, research in franchising, on the other hand, has recently been looking at how knowledge management can lead to successful franchise networks. Part of the studies on the topic analyzes contracts from the perspective of Agency Theory (AT), Transaction Costs Theory (TCT), Resource-Based View (RBV), InstitutionBased View (IBV) or even the Knowledge-Based View (KBV), a consequence or extension of the RBV. This study aims to comprehend the adequate theoretical basis for research on the definition and legal protection of knowledge in franchises, exploring the most common theoretical basis.
\end{abstract}

Keywords: knowledge; franchising; research; resources; theory.

Resumo: Pesquisas no âmbito das relações interorganizacionais apontam para o conhecimento como o recurso organizacional mais importante e, as pesquisas em franchising, por outro lado, há pouco tempo tem procurado entender como a gestão do conhecimento pode conduzir a redes de franquia de sucesso. Os estudos sobre o tema analisa os contratos sob a ótica da Teoria da Agência (TA), da Teoria dos Custos de Transação (TCT), da Visão Baseada em Recursos (VBR), da Visão Baseada nas Instituições (VBI), ou mesmo da Visão Baseada em Conhecimento (VBC), uma conseqüência ou extensão da VBR. O trabalho busca compreender as bases teóricas adequadas para a pesquisa sobre a definição e proteção jurídica do conhecimento em franquias, explorando as bases teóricas comuns ao estudo de franquias.

\footnotetext{
${ }^{1}$ Advogada, Mestre pelo Programa de Mestrado Profissional em Direito da Empresa e dos Negócios. Unisinos, Porto Alegre. E-mail: fernanda.larosa@baptistaluz.com.br

${ }^{2}$ Doutor, professor do Programa de Mestrado Profissional em Direito da Empresa e dos Negócios. Unisinos, Porto Alegre. E-mail: sibitencourt@unisinos.br
} 
Palavras-chave: conhecimento; franquias; pesquisa; recursos; teoria.

\section{INTRODUÇÃO}

A observação das relações de franquia formalizadas sob a vigência da antiga Lei de Franquias (Lei 8.955/95) sugere que, em que pese a lei tenha tido um papel bastante importante no que diz respeito à obrigação de revelação prévia de informações por parte das franqueadoras e na mitigação de riscos da relação, ela não conseguiu contemplar todos os contornos que permeiam a relação entre franqueado e franqueador e não abarca uma série de fatores que são importantes para o setor do franchising (Barroso, 2003), como, por exemplo, a conceituação, as formas de gestão e a proteção jurídica do know-how produzido e transmitido aos franqueados sob o contrato de franquia, seja no seu início, seja durante o curso da relação de franquia, até o seu término.

O franchising é objeto de estudo de variadas áreas, já tendo sido pesquisado sob as perspectivas de variadas ciências como o Empreendedorismo, o Marketing, a Economia, a Gestão Estratégica, o Direito, entre outros (Combs et al, 2004, p. 908). Se, por um lado, isto traz a vantagem de propiciar uma crescente e atualizada literatura sobre o tema, há também a desvantagem de cada uma dessas áreas de estudo, muitas vezes, adotar bases teóricas distintas, gerando certa confusão relativa aos resultados das pesquisas (Combs et al, 2004, p. 908).

Pesquisas no âmbito das relações interorganizacionais apontam para o conhecimento como o recurso organizacional mais importante e, as pesquisas em franchising, por outro lado, há pouco tempo tem procurado entender como a gestão do conhecimento pode conduzir a redes de franquia de sucesso. (Gorovaia, 2010, p. 235; Fu-Sheng et al., 2017, p. 98).

Por ora, é relevante referir que a criação e a transferência de recursos intangíveis em franchising, especialmente know-how e elementos de propriedade intelectual, não pode ser gerenciado no sentido tradicional de controle de fluxo de informações (Lindblom \& Tikkanen, 2010). Pelo contrário, o contrato de franquia usualmente deve encorajar a colaboração entre os integrantes da rede, franqueados e franqueador, para fins de criação e troca de conhecimentos, sendo, no entanto, necessário atentar para a preservação de direitos do franqueador relativamente à propriedade sobre alguns conhecimentos estratégicos produzidos. 
Diferentemente do que ocorre com patentes, marcas ou direitos de autor, as legislações não preveem direitos claros de propriedade sobre o conhecimento (Grant, 1996, p. 111).

Combs et al (2004) sugerem que a pesquisa em franchising pode se beneficiar de uma maior diversidade teórica, superando as bases teóricas usualmente utilizadas. Segundo os autores, a variedade teórica permite aos pesquisadores visualizar o mesmo fenômeno através de variadas lentes e, assim, construir um entendimento mais rico sobre o tema. Em artigo publicado em 2004, os autores identificaram que a Teoria da VBR era um campo ainda inexplorado nos estudos sobre franquias. Em artigo publicado em 2019, Iddy e Alon (2019) identificaram que novas pesquisas são necessárias para estabelecer variáveis específicas relacionadas ao conhecimento na literatura sobre franquias, indicando a gestão de conhecimento em franchising como uma agenda de pesquisa para o futuro.

Mesmo tendo sido identificado que o conhecimento é o elemento central da relação de franquia, parte dos estudos sobre o tema analisa os contratos sob a ótica da Teoria da Agência (TA), da Teoria dos Custos de Transação (TCT), da Visão Baseada em Recursos (VBR) ou mesmo da Visão Baseada nas Instituições (VBI).

Nesta direção, este trabalho procura compreender quais as bases teóricas adequadas para a pesquisa sobre a definição e proteção jurídica do conhecimento em franquias, explorando as bases teóricas comuns ao estudo de franquias e a partir de seus fundamentos e principais ideias se busca identificar a mais adequada para o desenvolvimento de futuras pesquisas sob a ótica do conhecimento gerado e transferido no âmbito da relação entre franqueador e franqueados.

\section{REFERENCIAL TEÓRICO}

\subsection{BASES TEÓRICAS COMUNS AO ESTUDO SOBRE FRANQUIAS}

\subsubsection{Teoria de Agência}

Conforme referido, a Teoria da Agência (TA) possui suas raízes na Teoria da Firma, desenvolvida por Ronald Coase no artigo seminal intitulado "The Nature of the Firm", publicado em 1937. Neste trabalho, Coase questiona o papel da firma em uma perspectiva neoclássica, argumentando que o sistema econômico não é controlado somente pelo mecanismo de preços. Nesse sentido, há, ao menos, dois mecanismos coordenados: no mercado, o sistema 
de preços indica a melhor alocação de recursos, e na firma, o empreendedor é responsável pela realocação de recursos (Coase 1937).

Sob a ótica da TA, o franchising, como um canal de distribuição, apresenta vantagens tais como a economia de escala, a padronização e o sistema vertical de marketing, além da busca por eficiência na operação da unidade franqueada por um proprietário (franqueado) e custos de monitoramento menores (Albuquerque et al., 2016, p. 554-565).

O franchising tem sido estudado a partir da TA, sendo que, em um apanhado da literatura, se destacam algumas incursões (Lafontaine et al, 1992; Hoffman et al, 2016; Madanoglu \& Castrogiovani, 2018; Jang \& Park, 2019).

Os autores LaFontaine et al (1992) fornecem uma avaliação empírica de várias explicações teóricas da agência para o franchising, incluindo o compartilhamento de riscos, o risco moral unilateral e o risco moral bilateral. LaFontaine et al (1992) destacam que modelos empíricos adotados usualmente neste campo de estudo adotam proxies para fatores como risco, risco moral e necessidade de capital dos franqueadores para explicar as decisões dos franqueadores sobre os termos de seus contratos (taxas de royalties e taxas de franquia) e sobre até que ponto eles usam o franchising como modelo de negócios.

Mais atualmente, Hoffman et al (2016) examinam como o ambiente institucional de um país afeta as atividades de expansão internacional das empresas franqueadoras nos Estados Unidos (EUA). Para este fim, Hoffman et al (2016) recorrem a teorias institucionais e de custos de transação para desenvolver um modelo e um conjunto de hipóteses sobre o efeito de instituições políticas, reguladoras e de infraestrutura, bem como instabilidade econômica, na expansão por franquias internacionais. Usando uma amostra de empresas franqueadoras dos EUA e dados de uma combinação de fontes secundárias, Hoffman et al (2016) testaram essas hipóteses e seus resultados demonstram pela primeira vez que, além da governança política favorável, o ambiente de negócios de um país, incluindo regulamentações de entrada, impostos e infraestrutura de comunicações, é um importante preditor da expansão das empresas de franquias estrangeiras naquele país.

No entendimento de Jang e Park (2019) para que um sistema de franquias obtenha sustentabilidade de longo prazo, equilibrar os benefícios entre franqueadores e franqueados é extremamente importante. A fim de expandir esta área de pesquisa, o estudo de Jang e Park 
(2019) revisou a literatura de franquia anterior e, em seguida, delineou o que é um sistema de franquias saudável. Além disso, este estudo também propôs um modelo para um relacionamento franqueador-franqueado sustentável. Ao desenvolver este modelo, Jang e Park (2019) sugerem que a qualidade do relacionamento é uma parte vital do sistema de franquias, além de ter identificado três componentes principais de uma relação franqueador-franqueado sustentável (ou seja, satisfação, confiança e comprometimento), bem como os antecedentes e as consequências do relacionamento. Conclusivamente, Jang e Park (2019) sugerem que os franqueados estão dispostos a continuar seu atual negócio de franquia somente quando estiverem satisfeitos com a imparcialidade, autonomia, formalização e suporte do franqueador.

Assim, o sistema de franquias, por um lado, reduz a necessidade de monitoramento dos agentes (franqueados), pois, uma vez que os franqueados são proprietários dos seus negócios, há um incentivo maior para que dediquem todos os seus esforços na boa administração das suas franquias. No entanto, há um risco de que busquem maximizar seus próprios interesses em detrimento dos interesses do franqueador, buscando, por exemplo, um aumento de lucros através da redução de custos nas suas atividades, o que pode prejudicar a imagem da marca e dos produtos ou serviços identificados com o franqueador e ser fonte originária de uma série de conflitos (Varotto \& Silva, 2017, p. 33).

A busca da maximização de utilidade provoca no agente o incentivo certo para a prática de ações que o levem ao melhor resultado financeiro, o que permite pressupor que o proprietário da franquia sempre buscará a maximização do seu resultado e, nesse viés, a figura do franqueado, como proprietário da unidade de negócio, substitui muito bem a figura de um funcionário da matriz na expansão de um negócio. Porém, não é possível assegurar que o agente sempre tomará a melhor decisão para os interesses do principal, o que faz surgir os custos de agência, que são aqueles custos relacionados à assimetria de informação e aos conflitos de interesse entre agente e principal (Jensen \& Meckling, 1976, p. 305-360). Os custos de agência podem ser (i) os custos de monitoramento pelo principal, relacionados à gestão da rede e ao acompanhamento da performance e do cumprimento das obrigações pelos agentes (ii) os custos do vínculo pelo agente, que consistem nos custos incorridos pelo agente para sinalizar seus esforços na observância dos limites ou restrições impostos à atividade do agente e, por fim, (iii) a perda residual, que consiste na criação de custo com impacto direto no lucro decorrente da 
divergência entre os interesses do principal e dos agentes, apesar dos custos de monitoramento e de vínculo (Jensen \& Meckling, 1976, p. 305-360).

\subsubsection{Teoria dos Custos de Transação}

A Teoria dos Custos de Transação (TCT) também tem sua origem nos estudos desenvolvidos por Ronald Coase e, posteriormente, recebeu contribuições significativas de Oliver Williamson. Segundo esta teoria, a busca de maior eficiência produtiva reflete-se nos padrões de conduta dos agentes e na forma pela qual as atividades econômicas são gerenciadas. Essa abordagem postula que as estruturas organizacionais, tal como a firma, o mercado, as hierarquias e os modelos híbridos ou contratos de longa duração, são o resultado da busca dos agentes econômicos pela minimização dos custos de transaçãp (Santos et al, 2017, p. 7).

Os custos de transação podem ser compreendidos como o dispêndio de recursos econômicos para planejar, adaptar e monitorar as interações entre os agentes, assegurando que o cumprimento das disposições contratuais ocorra de maneira satisfatória para as partes envolvidas e compatível com a sua funcionalidade econômica (Santos et al, 2017, p. 7). Dessa forma, pode-se dizer também que os custos de transação são os custos para a realização dos negócios e são, em sua maioria, difíceis de quantificar (Williamson, 1979).

Nesse sentido, o Teorema de Coase indica que as partes de um contrato elaborarão um acordo perfeito, ou seja, completo e eficiente, quando os custos de transação forem iguais a zero, porque, nesse caso, negociar condições adicionais não custará nada. Todavia, supor que não há quaisquer custos para realizar transações no mercado é bastante irrealista, pois, para que qualquer pessoa realize uma transação, será necessário identificar a outra parte, informar as condições sob as quais deseja negociar, conduzir as negociações, formular o respectivo contrato e monitorar o seu cumprimento (Coase, 1960). Os custos de transação variam conforme a natureza do contrato; quando os custos são muito altos em comparação com o excedente resultante da cooperação geram obstáculos à eficiência, ou, até mesmo, impedem a cooperação entre as partes (Cooter \& Ullen, 2010, p. 231).

A TCT, na versão de Oliver Williamson, se sustenta sobre dois pressupostos comportamentais, quais sejam, (i) a racionalidade limitada dos agentes econômicos; e (ii) o oportunismo presente nas ações dos agentes econômicos. A racionalidade limitada pressupõe que, mesmo que os indivíduos sejam racionais e busquem a maximização dos seus interesses, 
eles são limitados pela carência de informações, de modo que, ao invés de tomar uma decisão ótima, tomam apenas a decisão possível, considerando sua limitação de conhecimento. Todos os contratos complexos serão inevitavelmente incompletos dada a racionalidade limitada dos agentes que os elaboram (Williamson, 1979, p. 241).

Já o oportunismo, segundo Williamson, é o conceito central da TCT e se caracteriza como o comportamento de busca do interesse próprio com astúcia/malícia, sendo que nem todos os agentes apresentam este comportamento em mesmo grau. $\mathrm{O}$ conceito de oportunismo relaciona-se com a eficiência dos processos de informação entre as partes, pois decorre justamente da manipulação de assimetrias de informação (Williamson, 1979, p. 234). Com isso, surge a necessidade de ações de monitoramento e previsão de mecanismos contratuais aptos a evitar, ou ao menos minimizar, comportamentos negativos relacionados à omissão de informações das partes (Santos et al, 2017, p. 8).

Os autores Hendrikse et al (2015) fornecem um novo conceito teórico sobre completude contratual e a sua aplicação na incompletude de contratos de franquia, fundamentando-se nas perspectivas dos custos de transação e de governança relacional. Segundo os autores, os contratos de franquia possuem dois tipos de disposições: (i) direitos de decisão específicos, que detalham o que o franqueador e o que o franqueado devem fazer sob certas circunstâncias durante a vigência contratual, e (ii) direitos de decisão residual, que especificam os direitos do franqueador e do franqueado de tomar certas decisões durante a vigência contratual. A completude contratual, assim, é definida pela relação entre direitos de decisão específicos e residuais estipulados no contrato, sendo que o contrato de franquia será mais completo quanto mais direitos de decisão específicos e menos direitos de decisão residual houver. Defendem que os efeitos dos investimentos na transação sobre a completude contratual são influenciados pelo nível de dependência entre o franqueador e o franqueado: se houver dependência bilateral entre eles, os investimentos na transação têm um impacto negativo; se houver dependência unilateral, os investimentos possuem impacto positivo na completude contratual.

Os autores Gorovaia e Windsperger (2018) propõem uma combinação da TCT com a VBR para embasar a pesquisa realizada relativa à decisão dos franqueadores quanto ao tempo de duração dos contratos de franquia. Sustentam que essa decisão leva em consideração tanto aspectos relacionados à economia de custos de transação e mitigação de riscos, quanto aspectos 
relacionados à criação estratégica de valor através da alavancagem de recursos relacionados ao conhecimento, especialmente o know-how específico do franqueador e a sua marca, para fundamentar escolhas por contratos de longa duração.

A articulação entre a TCT e a VBR também é proposta por Santos (2017, p. 14), que defende que a firma seja compreendida como um conjunto de capacidades (conhecimentos e habilidades) que determina o que ela é capaz de fazer e permite o desenvolvimento de ativos específicos. A partir da percepção de que a firma é capaz de adquirir novos conhecimentos (recursos) e mudar, conforme seu interesse, sua estrutura de governança, ela desenvolve a capacidade de se modificar continuamente, o que garante a especificidade dos seus ativos ao longo do tempo, obtendo, assim, vantagem competitiva. Ainda segundo Santos (2017, p. 14), ambas teorias se relacionam com a busca de eficiência da firma, se diferenciando no que diz respeito ao modo de obtenção dessa eficiência - enquanto na TCT, a vantagem competitiva é obtida a partir da economia de custos de transação, na VBR, ocorre a partir de um conjunto de recursos e capacidades únicas.

\subsubsection{Visão Baseada nas Instituições}

Para Peng $(2002,2003)$ a Visão Baseada em Instituições (VBI) pode ser identificada como uma terceira perspectiva principal em gestão estratégica, sendo as duas primeiras as visões baseada na indústria que discute as condições e a extensão em que a indústria determina a estratégia e desempenho das firmas (Porter, 1980) e em recursos, que examina a ligação entre as características internas ou recursos das firmas e a implantação de estratégias que visam melhorar seu desemepnho (Barney, 1991).

Possibilita a superação das críticas de longa data da falta de atenção aos contextos das visões baseadas na indústria e nos recursos e contribui com novos discernimentos como parte do movimento intelectual mais amplo centrado no novo institucionalismo, uma abordagem com origem no trabalho de Meyer (1977) para o estudo de instituições que enfoca os efeitos restritivos e habilitadores de regras formais e informais sobre o comportamento de indivíduos e grupos. Em síntese, a VBI afirma que a eficiência de uma empresa (seja eficiência operacional, de gestão do conhecimento, ou de inovação etc.) depende, além de fatores internos, de fatores relacionados ao contexto onde opera. Estes fatores podem ser "recursos" (acesso a 
tecnologia, a mão de obra qualificada, suporte a projetos de inovação etc.) ou "instituições" (regras de jogo como políticas anticorrupção, propriedade intelectual, abertura a mercados internacionais etc.) (Peng, 2002; Peng \& Khoury, 2008; Lu, Tsang \& Peng, 2008).

Além disso, Lu, Tsang \& Peng (2008) observam que, até então, vinha sendo relativamente negligenciada em estudos sobre gestão do conhecimento e inovação, podendo desempenhar pelo menos três papéis nos processos neste contexto. Em primeiro lugar, o conhecimento deve ser percebido como legítimo e deve atender aos requisitos institucionais. Em segundo lugar, o conhecimento em si é, até certo ponto, dependente da instituição. Terceiro, a eficácia e eficiência da criação, transmissão e realocação de conhecimento são parcialmente determinadas pela infraestrutura institucional. Para Sun et al. (2015), um ambiente institucional inclui instituições formais, como o sistema jurídico, com leis, regras e regulamentos.

Em resposta a indagação de Meyer e Peng (2005), Peng (2001, 2003, 2009), Wright et al. (2005) e Yamakawa, Peng e Deeds (2008) para maior integração entre as visões institucionais e baseadas em recursos, Meyer et al. (2009) focalizaram um conceito central na direção da eficácia dos mercados em facilitar o acesso aos recursos buscados.

A VBI tem alcançado relevância no campo da estratégia e possibilitado diferentes aplicações, tais como em estudos de economias emergentes (Meyer et al., 2009), tendo como objeto o estudo de franquias (de Almeida, Lanfranchi \& Melo; 2018).

\subsubsection{Visão Baseada em Recursos}

A Visão Baseada em Recursos (VBR) funda-se nas ideias de Edith Penrose (1959) que descreveu a empresa como uma organização administrativa e um conjunto de recursos produtos, tanto físicos quanto humanos. Esta teoria busca explicar a diferenciação entre empresas e a vantagem de desempenho obtida por algumas empresas em relação a outras a partir da heterogeneidade organizacional. Dessa forma, seu principal fundamento é a compreensão do motivo pelo qual algumas empresas alcançam desempenho superior às outras. Para este fim, parte-se do pressuposto de que nem todos os recursos organizacionais são necessariamente estratégicos; a condição estratégica é atingida quando os recursos passam a ser portadores de diferenciais qualitativos positivos em relação aos seus concorrentes (Santos, 2017). Ou seja, o desempenho superior é alcançado quando os recursos organizacionais são arranjados e 
organizados pelas competências e capacidades desenvolvidas pelos gestores, transformando-os em ativos específicos, o que torna determinadas empresas mais eficientes do que suas competidoras (Grant, 1991).

Um "Recurso" pode ser entendido como tudo aquilo que pode ser considerado como uma força ou uma fraqueza de determinada empresa, ou, ainda, como todos os itens do patrimônio tangível ou intangível ligado à empresa de modo semipermanente. São recursos da empresa, exemplificativamente, as suas marcas, tecnologia in-house, os seus funcionários qualificados, contratos mercantis, maquinário, capital de giro, entre outros (Wernerfelt, 1984).

Segundo Grant (1996, p. 110), a VBR não é tanto uma teoria sobre a estrutura e comportamento das empresas sob o seu ponto de vista interno, podendo ser melhor compreendida como uma teoria que busca explicar e prever porque algumas empresas são capazes de estabelecer vantagens competitivas e, em assim fazendo, tornarem-se mais lucrativas. Dessa forma, a VBR enxerga a empresa como um agrupamento único de recursos e capacidades idiossincráticas, no qual a principal preocupação da administração deve ser a de maximizar valor a partir da exploração otimizada dos recursos e capacidades existentes, focando, também, no desenvolvimento das bases de recursos da empresa para o futuro.

Grant (1991, p. 116) defende, ainda, que os recursos e as capacidades da empresa devem representar a base para a definição da estratégia de longo prazo da empresa, isso porque, os recursos e capacidades internas fornecem a direção básica para a estratégia e, também, porque são a fonte primária das receitas e dos lucros da empresa. Quando uma empresa controla um recurso valioso e que é raro dentre os seus concorrentes, isso aumenta os lucros da empresa, e, assim, essa empresa possui vantagem competitiva, ainda que possa ser temporariamente. Essa vantagem se sustentará enquanto os concorrentes não a superarem por meio de imitação do recurso ou por encontrar substitutos estratégicos para o recurso (Barney, 1991)

Conforme já referido, a VBR também serviu como fundamento teórico para uma série de estudos sobre franchising (Welsh et al, 2011; Perrigot \& Pénard, 2013), especialmente relacionados ao campo da estratégia empresarial. Os autores enfatizam a importância da VBR para o estudo das estratégias de franqueadores, uma vez que os recursos intangíveis, como a marca, a reputação, o conceito de negócio e o know-how, exercem papel relevante para o sucesso no franchising (Perrigot \& Pénard, 2014, p. 111).

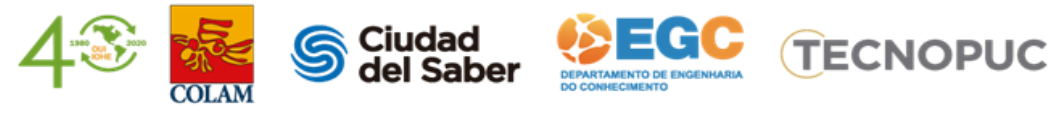


Nesse mesmo sentido, Gorovaia e Windsperger (2018) reforçam a relevância dos recursos intangíveis em franchising ao afirmar que a transferência de tais recursos dos franqueadores para os franqueados resulta em uma criação de valor conjunta e que requer colaboração de longo prazo entre os membros da rede, não devendo ser tratada pelo senso tradicional de simples fluxo de informações.

\subsubsection{Visão Baseada em Conhecimento}

A Visão Baseada em Conhecimento (VBC) tem sua origem com Robert Grant (1996). Segundo essa teoria, o conhecimento é o insumo estratégico para produção e fonte primária de valor para uma empresa (Grant, 1996, p. 112). A conexão entre a VBR e a VBC está na compreensão de que o conhecimento é um recurso empresarial. Nesta direção, a VBC pode ser vista como uma consequiência ou extensão da VBR, pois foca no conhecimento como o recurso mais estrategicamente importante de qualquer organização (Kogut \& Zander, 1992) e é um antecedente da inovação e desempenho da empresa (Darroch, 2005). Uma das principais proposições da VBC é de que as organizações existem para criar, transferir e transformar conhecimento em vantagem competitiva (Kogut \& Zander, 1992). A questão é que transferir conhecimento através das diversas áreas de uma empresa pode ser difícil e, ainda mais difícil através de uma rede de empresas independentes, unidas por relações contratuais apenas, como é o caso das redes de franquia.

Nesse sentido, é possível traçar uma relação da VBC com a TCT, que defende que surgirá uma firma, ou ocorrerá a ampliação das atividades de uma firma já existente, sempre que os custos administrativos da firma forem inferiores aos custos das transações de mercado que ela suplanta, ou, ainda, quando os ganhos que resultem da reorganização das atividades forem superiores aos custos da firma para organizá-los. Ou seja, as firmas existem para evitar os custos associados com as transações de mercado. Assim, a VBC volta sua atenção para os custos associados a um tipo específico de transação, quais sejam, aquelas envolvendo transferência de conhecimento (Grant, 1996, p. 113). A ênfase da teoria, portanto, se dá na empresa como uma gestora de produção de conhecimento, ao invés da visão clássica da empresa como uma gestora de transações (Grant, 1996, p. 113). 
O conhecimento, segundo a teoria, pode ser subjetivo ou objetivo, tácito ou explícito, procedimental ou declaratório, pessoal ou organizacional (Grant, 1996). Esse trabalho foca somente na distinção entre conhecimento tácito e explícito, porque essas características do conhecimento implicam em maior ou menor dificuldade na tangibilização do conhecimento e na definição de mecanismos adequados para sua transferência, conforme o tipo de conhecimento em questão, o que é relevante para franqueadores e franqueados.

O conhecimento explícito, por sua vez, é aquele que, por definição, está codificado e, por isso, é mais facilmente transferível; é majoritariamente composto de informação que está registrada em documentos ou sistemas de informação de cada empresa, como, por exemplo, as normas e procedimentos ligados a um processo específico empresarial (Nonaka, 1994). É revelado através da sua comunicação, o conhecimento tácito é revelado através da sua aplicação e adquirido pela prática (Grant, 1996, p. 111).

O conhecimento tácito reside nos indivíduos, em sua mente e suas habilidades, e, portanto, a sua transmissão depende da capacidade de transmissão e aprendizado de cada indivíduo (Nonaka \& Takeuchi, 1995). É do tipo mais difícil de codificar e transferir, pois se trata de conhecimento não verbalizado e intuitivo, que possui um caráter pessoal, que o torna mais complexo de comunicar e formalizar (Battistella et al, 2016).

Salienta-se que há desafios relacionados ao uso e transferência de ambos os tipos de conhecimento, pois, mesmo que o conhecimento esteja estruturado e codificado (explícito), é necessário, ainda, torná-lo acessível às pessoas que devem utilizá-lo, bem como garantir que as mesmas absorvam esse conhecimento (Battistella et al, 2016).

Cumpre ressaltar que, na prática, não existem formas puras de conhecimento tácito e explícito. Segundo Nonaka et al (2013, p. 3), todo o conhecimento está fundado em conhecimento tácito e, assim, até o mais explícito dos conhecimentos contém aspectos tácitos.

O conhecimento, como principal recurso de uma empresa, apresenta desafios também quanto à sua apropriabilidade. $\mathrm{O}$ conhecimento tácito não pode ser diretamente apropriado porque não pode ser diretamente transferido; o conhecimento explícito, por sua, vez pode ser apropriado somente através da sua aplicação em atividade produtiva (Grant, 1996, p. 111).

Nesse sentido, é importante a compreensão da empresa como uma organização dinâmica que deve constantemente criar e explorar novos conhecimentos. O aprendizado organizacional 
permanente permite que a empresa adquira, mude e preserve suas capacidades organizacionais e desenvolva a sua cultura organizacional.

Considerando a crescente relevância do conhecimento como recurso organizacional principal, Nonaka et al (2013) propõem um novo modelo organizacional batizado de “organização dinâmica fractal”, fundamentado na ideia da tríade do conhecimento, que consiste na integração e síntese do conhecimento tácito e explícito para fins de criar um terceiro tipo de conhecimento, denominado "phronesis". Esta tríade consiste em um processo espiral de conversão de conhecimento tácito e explícito e propicia a transformação e disseminação do conhecimento através da empresa e entre empresas.

Nesse aspecto, também é relevante apresentar o conceito de capacidade absortiva proposto por Cohen e Levinthal (1990, p. 128), caracterizado como a habilidade de uma empresa em avaliar, utilizar e explorar conhecimento obtido externamente. A capacidade absortiva se revela na habilidade da empresa em identificar o valor deste conhecimento primário exterior, assimilá-lo e aplicá-lo para fins comerciais. Em franchising, a capacidade absortiva é importante para medir a capacidade do franqueado em criar, armazenar, transformar, receber, replicar e aplicar o conhecimento (Iddy \& Alon, 2019).

\section{DISCUSSÃO}

Apresentados os principais elementos e conceitos da VBC, cumpre ressaltar que estudos recentes tem fundamentado na VBC a análise do franchising sob a ótica do conhecimento gerado e transferido no âmbito da relação, partindo da premissa que este conhecimento constitui o recurso primordial das empresas franqueadoras (Hussler \& Ronde, 2017; Gorovaia, 2010; FuSheng et al., 2017; Iddy \& Alon, 2019).

A perspectiva teórica da VBC permite analisar as relações que se desenvolvem entre franqueadores e franqueados por meio do contrato de franquia, especialmente no que diz respeito à transferência de conhecimento e, mais especificamente, de know-how. Segundo Gorovaia (2010, p. 235), a VBC tem o potencial de esclarecer como as fontes de conhecimento e as capacidades organizacionais podem aumentar a vantagem competitiva, criar valor e alavancar a performance de redes de franquias. Ainda, segundo Fu-Sheng (2017, p. 98), a VBC pode explicar como os franqueadores podem transferir seu conhecimento de forma mais simples 
e quais obstáculos podem encontrar na busca do desenvolvimento mútuo e no compartilhamento e simbiose das fontes de conhecimento trazidas também pelos franqueados do sistema de franquia.

De acordo com Hussler e Ronde (2017), por sua vez, a VBC pode fornecer contribuições quanto aos motivos que levam indivíduos a optarem por abrir um negócio franqueado, parte de uma rede, e não empreender de forma independente.

Por outro lado, as limitações da VBC estão relacionadas com a dificuldade de definir o caráter único e "difícil de imitar" de um determinado conhecimento apto a criar vantagem competitiva, bem como a dificuldade de mensurar o conhecimento empresarial (Iddy \& Alon, 2019, p. 772).

Do ponto de vista do franchising, esse determinado conhecimento apto a criar vantagem competitiva, usualmente, se caracteriza como o know-how do franqueador e a sua mensuração adquire ainda maior relevância, visto que a produção e transferência de know-how a terceiros interessados em ingressar na sua rede de franquias é a essência da atividade de uma franqueadora e fator chave de sucesso da rede (Paswan \& Wittmann, 2009; Guetta et al., 2013, p. 161; Gorovaia, 2010).

Adicionalmente, é possível perceber relações entre as diferentes bases teóricas exploradas neste estudo, como a que se estabelece entre a TCT e a TA e entre a TCT e a VBR, além da integração entre a VBI e a VBR e, ainda, a VBC vista como uma conseqüência ou extensão da VBR. Também, ao considerar a VBI como uma terceira perspectiva principal em gestão estratégica, seria possível conectar a VBR com a Indústria e com a própria VBI.

\section{CONSIDERAÇÕES FINAIS}

Ao se explorar as bases teóricas comuns ao estudo de franquias e a partir de seus fundamentos e principais ideias não é possível definir a mais adequada para o desenvolvimento de futuras pesquisas sob a ótica do conhecimento gerado e transferido no âmbito da relação entre franqueador e franqueados.

A VBC apresenta-se como a base teórica de maior utilidade para a pesquisa sobre a definição e proteção jurídica do conhecimento gerado e transferido no âmbito da relação entre franqueador e franqueados, dada a natureza estratégica e relevante dos recursos intangíveis em 
franchising. Parte da premissa de que o conhecimento é o recurso essencial para o sucesso em franquias, o que passa por uma adequada proteção jurídica, tanto legal quanto contratual.

Entretanto, não se apregoa uma limitação em torno da VBC. Neste caso, a proteção jurídica do conhecimento em franquias, pode (e deve) ser explorada por diversas teorias e suas combinações, as quais permitam "olhares" do mesmo fenômeno desde diferentes perspectivas.

Aliás, esta é uma das limitações deste estudo, pois não são exploradas em profundidade as combinações identificadas entre as bases teóricas exploradas. Outra limitação diz respeito a oportunidade de adoção de bases teóricas oriundas de outras áreas, que não as tratadas neste estudo e mais diretamente associadas ao franchising como objeto de estudo, entre as quais as ligadas a sociologia por exemplo.

Como oportunidades para pesquisas futuras, além da adoção das bases teóricas mais próximas ao franchising aqui exploradas em estudos teórico-empíricos, o aprofundamento do entendimento destas bases teóricas exploradas e suas combinações em associação com franchising, tem o potencial de gerar novas e instigantes reflexões em aspectos que possam ir além da definição e proteção jurídica do conhecimento em franquias.

\section{REFERÊNCIAS}

Akerlof, G. A. (1978). The market for "lemons": Quality uncertainty and the market mechanism. In Uncertainty in economics (pp. 235-251). Academic Press.

Albuquerque, M., Ladeira, R., \& Larocca, M. T. (2016). Conflitos potenciais na relação entre franqueadores e franqueados soteropolitanos. Revista Brasileira de Marketing, 15(4), 554-565.

Barney, J. (1991). Firm resources and sustained competitive advantage. Journal of management, 17(1), 99-120.

Barroso, L. F. (2003). Franchising: modificações à lei vigente: estratégia e gestão. Editora Forense.

Battistella, C., De Toni, A. F., \& Pillon, R. (2016). Inter-organisational technology/knowledge transfer: a framework from critical literature review. The Journal of Technology Transfer, 41(5), 1195-1234.

Coase, R. (2009). O problema do custo social. The Latin American and Caribbean journal of legal studies, 3(1), 9.

Coase, R. H. (1995). The nature of the firm. In Essential readings in economics (pp. 37-54). Palgrave, London.

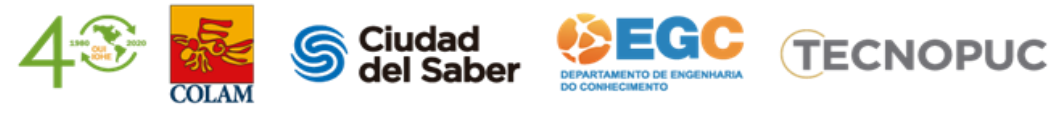


Cohen, W. M., \& Levinthal, D. A. (1990). Absorptive capacity: A new perspective on learning and innovation. Administrative science quarterly, 128-152.

Combs, J. G., Michael, S. C., \& Castrogiovanni, G. J. (2004). Franchising: A review and avenues to greater theoretical diversity. Journal of management, 30(6), 907-931.

Darroch, J. (2005). Knowledge management, innovation and firm performance. Journal of Knowledge Management, 9(3), 101.

de Almeida, M. D. J. F., Lanfranchi, A. G., \& Melo, P. L. D. R. (2018). Legal environment of the destination countries of internationalized Brazilian franchise chains. Review of International Business, São Paulo, 13(3), 14-27.

de Melo Santos, N., Ferraz, I. N., Falqueto, J. M. Z., \& Verga, E. (2017). A Teoria dos Custos de Transação nas Pesquisas de Estratégia no Brasil. Revista Ibero Americana de Estratégia, 16(2), 4-18.

Gorovaia, N., \& Windsperger, J. (2010). The use of knowledge transfer mechanisms in franchising. Knowledge and Process Management, 17(1), 12-21.

Gorovaia, N., \& Windsperger, J. (2018). The choice of contract duration in franchising networks: A transaction cost and resource-based view. Industrial Marketing Management, 75, 125-133.

Grant, R. M. (1991). The resource-based theory of competitive advantage: implications for strategy formulation. California management review, 33(3), 114-135.

Grant, R. M. (1996). Toward a knowledge-based theory of the firm. Strategic management journal, 17(S2), 109-122.

Guetta, A., Von Jess, A. C., Godiño, D., Schwartz, J., Monteiro, J., Barroso, L. F., ... \& Ayres, R. (2013). Franchising: Aprenda com os especialistas. Prime Books Brasil.

Hendrikse, G., Hippmann, P., \& Windsperger, J. (2015). Trust, transaction costs and contractual incompleteness in franchising. Small Business Economics, 44(4), 867-888.

Hoffman, R. C., Munemo, J., \& Watson, S. (2016). International franchise expansion: the role of institutions and transaction costs. Journal of International Management, 22(2), 101114.

Hussler, C., \& Ronde, P. (2015). To be or not to be franchised? A knowledge-based perspective. Knowledge Management Research \& Practice, 13(4), 429-445.

Iddy, J. J., \& Alon, I. (2019). Knowledge management in franchising: a research agenda. Journal of Knowledge Management.

Jang, S. S., \& Park, K. (2019). A sustainable franchisor-franchisee relationship model: Toward the franchise win-win theory. International Journal of Hospitality Management, 76, 1324.

Jensen, M. C., \& Meckling, W. H. (1976). Theory of the firm: Managerial behavior, agency costs and ownership structure. Journal of financial economics, 3(4), 305-360. LAFONTAINE, Francine et al. Agency theory and franchising: some empirical results. RAND journal of Economics, v. 23, n. 2, p. 263-283, 1992.

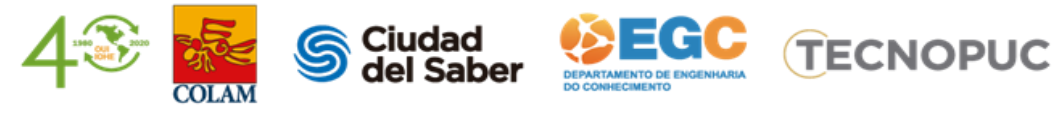


Lamy, M. (2002). Franquia pública. J. de Oliveira.

Lindblom, A., \& Tikkanen, H. (2010). Knowledge creation and business format franchising. Management Decision.

Lu, Y., Tsang, E. W., \& Peng, M. W. (2008). Knowledge management and innovation strategy in the Asia Pacific: Toward an institution-based view.

Madanoglu, M., \& Castrogiovanni, G. J. (2018). Franchising proportion and network failure. Small Business Economics, 50(4), 697-715.

Meyer, J. W., \& Rowan, B. (1977). Institutionalized organizations: Formal structure as myth and ceremony. American journal of sociology, 83(2), 340-363.

Meyer, K. E., \& Peng, M. W. (2005). Probing theoretically into Central and Eastern Europe: Transactions, resources, and institutions. Journal of international business studies, 36(6), 600-621.

Meyer, K. E., Estrin, S., Bhaumik, S. K., \& Peng, M. W. (2009). Institutions, resources, and entry strategies in emerging economies. Strategic management journal, 30(1), 61-80.

Nonaka, I. (1994). A dynamic theory of organizational knowledge creation. Organization science, 5(1), 14-37.

Nonaka, I., \& Takeuchi, H. (1995). The knowledge-creating company: How Japanese companies create the dynamics of innovation. Oxford university press.

Nonaka, I., Kodama, M., Hirose, A., \& Kohlbacher, F. (2014). Dynamic fractal organizations for promoting knowledge-based transformation-A new paradigm for organizational theory. European Management Journal, 32(1), 137-146.

Peng, M. W. (2001). The resource-based view and international business. Journal of management, 27(6), 803-829.

Peng, M. W. (2002). Towards an institution-based view of business strategy. Asia Pacific Journal of Management, 19(2-3), 251-267.

Peng, M. W. (2003). Institutional transitions and strategic choices. Academy of management review, 28(2), 275-296.

Peng, M. W., \& Khoury, T. A. (2008). Unbundling the institution-based view of international business strategy. In The Oxford handbook of international business.

Peng, M. W., Sun, S. L., Pinkham, B., \& Chen, H. (2009). The institution-based view as a third leg for a strategy tripod. Academy of Management Perspectives, 23(3), 63-81.

Penrose, E., \& Penrose, E. T. (2009). The Theory of the Growth of the Firm. Oxford university press.

Perrigot, R., \& Pénard, T. (2013). Determinants of e-commerce strategy in franchising: A resource-based view. International Journal of Electronic Commerce, 17(3), 109-130.

Porter, M. E., \& Strategy, C. (1980). Techniques for analyzing industries and competitors. Competitive Strategy. New York: Free.

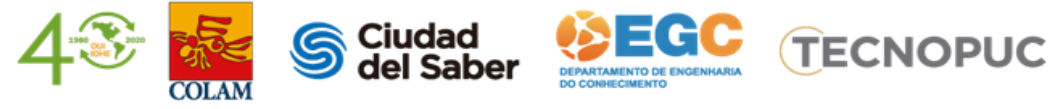


Sun, S. L., Peng, M. W., Lee, R. P., \& Tan, W. (2015). Institutional open access at home and outward internationalization. Journal of World Business, 50(1), 234-246.

Varotto, L. F., \& Silva, L. A. (2017). Evolution in franchising: Trends and new perspectives. Revista Eletrônica de Negócios Internacionais: Internext, 12(3), 31-42.

Welsh, D. H., Davis, A. E., Desplaces, D. E., \& Falbe, C. M. (2011). A resource-based view of three forms of business in the startup phase: Implications for franchising. Journal of Small Business Strategy, 22(1), 47-66.

Wernerfelt, B. (1984). A resource-based view of the firm. Strategic management journal, 5(2), 171-180.

Williamson, O. E. (1979). Transaction-cost economics: the governance of contractual relations. The journal of Law and Economics, 22(2), 233-261.

Wright, M., Filatotchev, I., Hoskisson, R. E., \& Peng, M. W. (2005). Strategy research in emerging economies: Challenging the conventional wisdom. Journal of management studies, 42(1), 1-33.

Yamakawa, Y., Peng, M. W., \& Deeds, D. L. (2008). What drives new ventures to internationalize from emerging to developed economies?. Entrepreneurship theory and practice, 32(1), 59-82. 